\title{
Brouwer Fixed Point Theorem for Simplexes
}

\author{
Karol Pąk \\ Institute of Informatics \\ University of Białystok \\ Poland
}

\begin{abstract}
Summary. In this article we prove the Brouwer fixed point theorem for an arbitrary simplex which is the convex hull of its $n+1$ affinely indepedent vertices of $\mathcal{E}^{n}$. First we introduce the Lebesgue number, which for an arbitrary open cover of a compact metric space $\mathfrak{M}$ is a positive real number so that any ball of about such radius must be completely contained in a member of the cover. Then we introduce the notion of a bounded simplicial complex and the diameter of a bounded simplicial complex. We also prove the estimation of diameter decrease which is connected with the barycentric subdivision. Finally, we prove the Brouwer fixed point theorem and compute the small inductive dimension of $\mathcal{E}^{n}$. This article is based on [16].
\end{abstract}

MML identifier: SIMPLEX2, version: $7.11 .07 \quad 4.160 .1126$

The papers [7], [31], [1], [8], [11], [17], [30], [14], [20], [4], [13], [9], [32], [21], [5], [19], [2], [3], [6], [22], [24], [18], [35], [26], [29], [33], [23], [27], [28], [34], [15], [25], $[12]$, and [10] provide the terminology and notation for this paper.

\section{The Lebesgue Number}

In this paper $M$ is a non empty metric space and $F, G$ are open families of subsets of $M_{\text {top }}$.

Let us consider $M$. Let us assume that $M_{\text {top }}$ is compact. Let $F$ be a family of subsets of $M_{\text {top }}$. Let us assume that $F$ is open and $F$ is a cover of $M_{\text {top }}$. A positive real number is said to be a Lebesgue number of $F$ if:

(Def. 1) For every point $p$ of $M$ there exists a subset $A$ of $M_{\text {top }}$ such that $A \in F$ and $\operatorname{Ball}(p$, it $) \subseteq A$. 
In the sequel $L$ denotes a Lebesgue number of $F$.

Next we state three propositions:

(1) If $M_{\text {top }}$ is compact and $F$ is a cover of $M_{\text {top }}$ and $F \subseteq G$, then $L$ is a Lebesgue number of $G$.

(2) If $M_{\text {top }}$ is compact and $F$ is a cover of $M_{\text {top }}$ and finer than $G$, then $L$ is a Lebesgue number of $G$.

(3) Let $L_{1}$ be a positive real number. Suppose $M_{\text {top }}$ is compact and $F$ is a cover of $M_{\text {top }}$ and $L_{1} \leq L$. Then $L_{1}$ is a Lebesgue number of $F$.

\section{Bounded Simplicial Complexes}

In the sequel $n, k$ denote natural numbers, $X$ denotes a set, and $K$ denotes a simplicial complex structure.

Let us consider $M$. One can check that every subset of $M$ which is finite is also bounded.

Next we state the proposition

(4) For every finite non empty subset $S$ of $M$ there exist points $p, q$ of $M$ such that $p, q \in S$ and $\rho(p, q)=\varnothing S$.

Let us consider $M, K$. We say that $K$ is $M$-bounded if and only if:

(Def. 2) There exists $r$ such that for every $A$ such that $A \in$ the topology of $K$ holds $A$ is bounded and $\varnothing A \leq r$.

The following proposition is true

(5) Let $K$ be a non void simplicial complex structure. If $K$ is $M$-bounded and $A$ is a simplex of $K$, then $A$ is bounded.

Let us consider $M, X$. Note that there exists a simplicial complex of $X$ which is $M$-bounded and non void.

Let us consider $M$. Note that there exists a simplicial complex structure which is $M$-bounded, non void, subset-closed, and finite-membered.

Let us consider $M, X$ and let $K$ be an $M$-bounded simplicial complex str of $X$. Note that every sub simplicial complex of $K$ is $M$-bounded.

Let us consider $M, X$, let $K$ be an $M$-bounded subset-closed simplicial complex str of $X$, and let $i$ be an integer. One can verify that the skeleton of $K$ and $i$ is $M$-bounded.

The following proposition is true

(6) If $K$ is finite-vertices, then $K$ is $M$-bounded. 


\section{The Diameter of a Bounded Simplicial Complex}

Let us consider $M$ and let $K$ be a simplicial complex structure. Let us assume that $K$ is $M$-bounded. The functor diameter $(M, K)$ yielding a real number is defined by:

(Def. 3)(i) For every $A$ such that $A \in$ the topology of $K$ holds $\varnothing A \leq$ diameter $(M, K)$ and for every $r$ such that for every $A$ such that $A \in$ the topology of $K$ holds $\varnothing A \leq r$ holds $r \geq \operatorname{diameter}(M, K)$ if the topology of $K$ meets $2^{\Omega_{M}}$,

(ii) $\operatorname{diameter}(M, K)=0$, otherwise.

One can prove the following three propositions:

(7) If $K$ is $M$-bounded, then $0 \leq \operatorname{diameter}(M, K)$.

(8) For every $M$-bounded simplicial complex str $K$ of $X$ and for every sub simplicial complex $K_{1}$ of $K$ holds diameter $\left(M, K_{1}\right) \leq \operatorname{diameter}(M, K)$.

(9) Let $K$ be an $M$-bounded subset-closed simplicial complex str of $X$ and $i$ be an integer. Then $\operatorname{diameter}(M$, the skeleton of $K$ and $i) \leq$ diameter $(M, K)$.

Let us consider $M$ and let $K$ be an $M$-bounded non void subset-closed simplicial complex structure. Then $\operatorname{diameter}(M, K)$ is a real number and it can be characterized by the condition:

(Def. 4)(i) For every $A$ such that $A$ is a simplex of $K$ holds $\varnothing A \leq$ diameter $(M, K)$, and

(ii) for every $r$ such that for every $A$ such that $A$ is a simplex of $K$ holds $\varnothing A \leq r$ holds $r \geq \operatorname{diameter}(M, K)$.

Next we state the proposition

(10) For every finite subset $S$ of $M$ holds diameter $(M$, the complex of $\{S\})=$ $\varnothing S$.

Let us consider $n$ and let $K$ be a simplicial complex $\operatorname{str}$ of $\mathcal{E}_{\mathrm{T}}^{n}$. We say that $K$ is bounded if and only if:

(Def. 5) $K$ is $\mathcal{E}^{n}$-bounded.

The functor $\varnothing K$ yielding a real number is defined as follows:

(Def. 6) $\varnothing K=\operatorname{diameter}\left(\mathcal{E}^{n}, K\right)$.

Let us consider $n$. One can verify the following observations:

* every simplicial complex str of $\mathcal{E}_{\mathrm{T}}^{n}$ which is bounded is also $\mathcal{E}^{n}$-bounded,

* there exists a simplicial complex of $\mathcal{E}_{\mathrm{T}}^{n}$ which is bounded, affinely independent, simplex-join-closed, non void, finite-degree, and total, and

* every simplicial complex $\operatorname{str}$ of $\mathcal{E}_{\mathrm{T}}^{n}$ which is finite-vertices is also bounded. 


\section{The Estimation of Diameter of the Barycentric Subdivision}

In the sequel $V$ is a real linear space.

The following two propositions are true:

(11) Let $S$ be a simplex of BCS $K_{2}$ and $F$ be a $\subseteq$-linear finite finite-membered family of subsets of $V$. Suppose $S=(\text { the center of mass } V)^{\circ} F$ and $\bigcup F$ is a simplex of $K_{2}$. Let $a_{1}, a_{2}$ be vectors of $V$. Suppose $a_{1}, a_{2} \in S$. Then there exist vectors $b_{1}, b_{2}$ of $V$ and there exists a real number $r$ such that $b_{1} \in$ Vertices BCS (the complex of $\{\bigcup F\}$ ) and $b_{2} \in$ Vertices BCS (the complex of $\{\bigcup F\})$ and $a_{1}-a_{2}=r \cdot\left(b_{1}-b_{2}\right)$ and $0 \leq r \leq \frac{\overline{\overline{U F}}-1}{\overline{\overline{U F}}}$.

(12) Let $A$ be an affinely independent subset of $\mathcal{E}_{\mathrm{T}}^{n}$ and $E$ be an enumeration of $A$. If $\operatorname{dom} E \backslash X$ is non empty, then conv $E^{\circ} X=\bigcap\{\operatorname{conv} A \backslash\{E(k)\} ; k$ ranges over elements of $\mathbb{N}: k \in \operatorname{dom} E \backslash X$ \}.

In the sequel $A$ denotes a subset of $\mathcal{E}_{\mathrm{T}}^{n}$.

The following three propositions are true:

(13) For every bounded subset $a$ of $\mathcal{E}^{n}$ such that $a=A$ and for every point $p$ of $\mathcal{E}^{n}$ such that $p \in \operatorname{conv} A$ holds conv $A \subseteq \overline{\operatorname{Ball}}(p, \varnothing a)$.

(14) $A$ is Bounded iff conv $A$ is Bounded.

(15) For all bounded subsets $a, c_{1}$ of $\mathcal{E}^{n}$ such that $c_{1}=\operatorname{conv} A$ and $a=A$ holds $\varnothing a=\varnothing c_{1}$.

Let us consider $n$ and let $K$ be a bounded simplicial complex $\operatorname{str}$ of $\mathcal{E}_{\mathrm{T}}^{n}$.

Observe that every subdivision str of $K$ is bounded.

The following propositions are true:

(16) For every bounded finite-degree non void simplicial complex $K$ of $\mathcal{E}_{\mathrm{T}}^{n}$ such that $|K| \subseteq \Omega_{K}$ holds $\varnothing$ BCS $K \leq \frac{\operatorname{degree}(K)}{\operatorname{degree}(K)+1} \cdot \varnothing K$.

(17) For every bounded finite-degree non void simplicial complex $K$ of $\mathcal{E}_{\mathrm{T}}^{n}$ such that $|K| \subseteq \Omega_{K}$ holds $\varnothing \operatorname{BCS}(k, K) \leq\left(\frac{\text { degree }(K)}{\operatorname{degree}(K)+1}\right)^{k} \cdot \varnothing K$.

(18) Let $K$ be a bounded finite-degree non void simplicial complex of $\mathcal{E}_{\mathrm{T}}^{n}$. If $|K| \subseteq \Omega_{K}$, then for every $r$ such that $r>0$ there exists $k$ such that $\varnothing \operatorname{BCS}(k, K)<r$.

(19) Let $i, j$ be elements of $\mathbb{N}$. Then there exists a function $f$ from $\mathcal{E}_{\mathrm{T}}^{i} \times \mathcal{E}_{\mathrm{T}}^{j}$ into $\mathcal{E}_{\mathrm{T}}^{i+j}$ such that $f$ is homeomorphism and for every element $f_{1}$ of $\mathcal{E}_{\mathrm{T}}^{i}$ and for every element $f_{2}$ of $\mathcal{E}_{\mathrm{T}}^{j}$ holds $f\left(f_{1}, f_{2}\right)=f_{1} \frown f_{2}$.

(20) Let $i, j$ be elements of $\mathbb{N}$ and $f$ be a function from $\mathcal{E}_{\mathrm{T}}^{i} \times \mathcal{E}_{\mathrm{T}}^{j}$ into $\mathcal{E}_{\mathrm{T}}^{i+j}$. Suppose that for every element $f_{1}$ of $\mathcal{E}_{\mathrm{T}}^{i}$ and for every element $f_{2}$ of $\mathcal{E}_{\mathrm{T}}^{j}$ holds $f\left(f_{1}, f_{2}\right)=f_{1} \frown f_{2}$. Let given $r, f_{1}$ be a point of $\mathcal{E}^{i}, f_{2}$ be a point of $\mathcal{E}^{j}$, and $f_{3}$ be a point of $\mathcal{E}^{i+j}$. If $f_{3}=f_{1} \frown f_{2}$, then $f^{\circ}$ (OpenHypercube $\left(f_{1}, r\right) \times$ OpenHypercube $\left.\left(f_{2}, r\right)\right)=$ OpenHypercube $\left(f_{3}, r\right)$. 
(21) $A$ is Bounded iff there exists a point $p$ of $\mathcal{E}^{n}$ and there exists $r$ such that $A \subseteq$ OpenHypercube $(p, r)$.

Let us consider $n$. Observe that every subset of $\mathcal{E}_{\mathrm{T}}^{n}$ which is closed and Bounded is also compact.

Let us consider $n$ and let $A$ be an affinely independent subset of $\mathcal{E}_{\mathrm{T}}^{n}$. One can verify that conv $A$ is compact.

\section{MAIN Theorems}

Next we state the proposition

(22) Let $A$ be a non empty affinely independent subset of $\mathcal{E}_{\mathrm{T}}^{n}, E$ be an enumeration of $A$, and $F$ be a finite sequence of elements of $2^{\text {the carrier of } \mathcal{E}_{\mathrm{T}}^{n}\lceil\operatorname{conv} A}$. Suppose len $F=\overline{\bar{A}}$ and $\operatorname{rng} F$ is closed and for every subset $S$ of $\operatorname{dom} F$ holds conv $E^{\circ} S \subseteq \bigcup\left(F^{\circ} S\right)$. Then $\bigcap \operatorname{rng} F$ is non empty.

In the sequel $A$ denotes an affinely independent subset of $\mathcal{E}_{\mathrm{T}}^{n}$.

Next we state four propositions:

(23) Let given $A$. Suppose $\overline{\bar{A}}=n+1$. Let $f$ be a continuous function from $\mathcal{E}_{\mathrm{T}}^{n}\left\lceil\operatorname{conv} A\right.$ into $\mathcal{E}_{\mathrm{T}}^{n}\left\lceil\operatorname{conv} A\right.$. Then there exists a point $p$ of $\mathcal{E}_{\mathrm{T}}^{n}$ such that $p \in \operatorname{dom} f$ and $f(p)=p$.

(24) For every $A$ such that $\overline{\bar{A}}=n+1$ holds every continuous function from $\mathcal{E}_{\mathrm{T}}^{n}\left\lceil\operatorname{conv} A\right.$ into $\mathcal{E}_{\mathrm{T}}^{n}\lceil\operatorname{conv} A$ has a fixpoint.

(25) If $\overline{\bar{A}}=n+1$, then ind conv $A=n$.

(26) $\operatorname{ind}\left(\mathcal{E}_{\mathrm{T}}^{n}\right)=n$.

\section{REFERENCES}

[1] Grzegorz Bancerek. Cardinal numbers. Formalized Mathematics, 1(2):377-382, 1990.

[2] Grzegorz Bancerek. The fundamental properties of natural numbers. Formalized Mathematics, 1(1):41-46, 1990.

[3] Grzegorz Bancerek. The ordinal numbers. Formalized Mathematics, 1(1):91-96, 1990.

[4] Grzegorz Bancerek and Krzysztof Hryniewiecki. Segments of natural numbers and finite sequences. Formalized Mathematics, 1(1):107-114, 1990.

[5] Grzegorz Bancerek and Yasunari Shidama. Introduction to matroids. Formalized Mathematics, 16(4):325-332, 2008, doi:10.2478/v10037-008-0040-0.

[6] Leszek Borys. Paracompact and metrizable spaces. Formalized Mathematics, 2(4):481485, 1991

[7] Czesław Byliński. Binary operations. Formalized Mathematics, 1(1):175-180, 1990.

[8] Czesław Byliński. The complex numbers. Formalized Mathematics, 1(3):507-513, 1990.

[9] Czesław Byliński. Functions and their basic properties. Formalized Mathematics, 1(1):5565, 1990.

[10] Czesław Byliński. Some basic properties of sets. Formalized Mathematics, 1(1):47-53, 1990.

[11] Agata Darmochwał. Compact spaces. Formalized Mathematics, 1(2):383-386, 1990.

[12] Agata Darmochwał. Families of subsets, subspaces and mappings in topological spaces. Formalized Mathematics, 1(2):257-261, 1990

[13] Agata Darmochwał. Finite sets. Formalized Mathematics, 1(1):165-167, 1990.

[14] Agata Darmochwał. The Euclidean space. Formalized Mathematics, 2(4):599-603, 1991. 
[15] Alicia de la Cruz. Totally bounded metric spaces. Formalized Mathematics, 2(4):559-562, 1991.

[16] Roman Duda. Wprowadzenie do topologii. PWN, 1986.

[17] Noboru Endou, Takashi Mitsuishi, and Yasunari Shidama. Convex sets and convex combinations. Formalized Mathematics, 11(1):53-58, 2003.

[18] Krzysztof Hryniewiecki. Basic properties of real numbers. Formalized Mathematics, 1(1):35-40, 1990.

[19] Stanisława Kanas, Adam Lecko, and Mariusz Startek. Metric spaces. Formalized Mathematics, 1(3):607-610, 1990.

[20] Artur Korniłowicz. The correspondence between $n$-dimensional Euclidean space and the product of $n$ real lines. Formalized Mathematics, 18(1):81-85, 2010, doi: 10.2478/v10037010-0011-0.

[21] Yatsuka Nakamura, Andrzej Trybulec, and Czesław Byliński. Bounded domains and unbounded domains. Formalized Mathematics, 8(1):1-13, 1999.

[22] Adam Naumowicz. On Segre's product of partial line spaces. Formalized Mathematics, 9(2):383-390, 2001.

[23] Beata Padlewska. Families of sets. Formalized Mathematics, 1(1):147-152, 1990.

[24] Beata Padlewska and Agata Darmochwał. Topological spaces and continuous functions. Formalized Mathematics, 1(1):223-230, 1990.

[25] Karol Pąk. Small inductive dimension of topological spaces. Formalized Mathematics, 17(3):207-212, 2009, doi: 10.2478/v10037-009-0025-7.

[26] Karol Pąk. Affine independence in vector spaces. Formalized Mathematics, 18(1):87-93, 2010, doi: 10.2478/v10037-010-0012-z.

[27] Karol Pąk. Abstract simplicial complexes. Formalized Mathematics, 18(1):95-106, 2010, doi: 10.2478/v10037-010-0013-y.

[28] Karol Pąk. Sperner's lemma. Formalized Mathematics, 18(4):189-196, 2010, doi: 10.2478/v10037-010-0022-x.

[29] Karol Pąk. Continuity of barycentric coordinates in Euclidean topological spaces. Formalized Mathematics, 19(3):139-144, 2011, doi: 10.2478/v10037-011-0022-5.

[30] Andrzej Trybulec. Domains and their Cartesian products. Formalized Mathematics, 1(1):115-122, 1990.

[31] Andrzej Trybulec. A Borsuk theorem on homotopy types. Formalized Mathematics, 2(4):535-545, 1991.

[32] Michał J. Trybulec. Integers. Formalized Mathematics, 1(3):501-505, 1990.

[33] Wojciech A. Trybulec. Vectors in real linear space. Formalized Mathematics, 1(2):291-296, 1990.

[34] Zinaida Trybulec. Properties of subsets. Formalized Mathematics, 1(1):67-71, 1990.

[35] Edmund Woronowicz. Relations defined on sets. Formalized Mathematics, 1(1):181-186, 1990. 